\title{
Analysis of variations in diesel engine idle vibration
}

M Ajovalasit* and $\mathbf{J}$ Giacomin

Department of Mechanical Engineering, The University of Sheffield, Sheffield, UK

\begin{abstract}
The variations in diesel engine idle vibration caused by fuels of different composition and their contributions to the variations in steering wheel vibrations were assessed. The time-varying covariance method (TV-AutoCov) and time-frequency continuous wavelet transform (CWT) techniques were used to obtain the cyclic and instantaneous characteristics of the vibration data acquired from two turbocharged four-cylinder, four-stroke diesel engine vehicles at idle under 12 different fuel conditions. The analysis revealed that TV-AutoCov analysis was the most effective for detecting changes in cycle-to-cycle combustion energy (22.61 per cent), whereas changes in the instantaneous values of the combustion peaks were best measured using the CWT method ( 2.47 per cent). On the other hand, both methods showed that diesel idle vibration was more affected by amplitude modulation (12.54 per cent) than frequency modulation (4.46 per cent). The results of this work suggest the use of amplitude modulated signals for studying the human subjective response to diesel idle vibration at the steering wheel in passenger cars.
\end{abstract}

Keywords: diesel, fuel, idle vibration, steering wheel, human perception, wavelet analysis

\section{NOTATION}

$a$

$b$

$\mathrm{CCV}$

$\mathrm{CN}$

$\mathrm{CP}$

$\mathrm{CV}$

CVCE

$\mathrm{CVCF}$

CVIA

CVIF

CWT

$f_{\mathrm{s}}$

$f_{x}$

$f_{0}$

FFT

$F_{\mathrm{F}}$

$F_{\text {REV }}$

$\mathrm{i}$ sale dilation

translation parameter

cycle-to-cycle variation

cetane number

cycle period

coefficient of variation

coefficient of variation in

cycle-to-cycle energy

coefficient of variation in

cycle-to-cycle frequency

coefficient of variation in

instantaneous amplitude

coefficient of variation in

instantaneous frequency

continuous wavelet transform

sampling frequency of the signal

frequency of the signal

frequency of oscillation of the

wavelet

fast Fourier transform

firing frequency

revolution frequency of crankshaft

$\sqrt{-1}$ imaginary unit $k$

$j$

$m$

$\mathrm{m} / \mathrm{s}^{2}$

$N$

$\mathrm{NVH}$

OWT

PSD

$\mathrm{r} / \mathrm{min}$

r.m.s.

$R_{x}$

$\mathrm{S}$

SD

STFT

$t$

TV-AutoCov

TW

$T_{\mathrm{F}}$

WT

WVD

$W_{\psi}(a, b)$

$x(t)$

$\bar{x}$

$\beta$

$\Delta f$

$\psi(t)$

$\psi_{a, b}(t)$

number of wavelet coefficients wavelet level

number of fuel tested

acceleration

sample number

noise, vibration and harshness

orthogonal wavelet transform

power spectral density

revolution per minute

root mean square

autocovariance function

second

standard deviation

short-time Fourier transform

time

time-varying autocovariance

time sliding window

firing period

wavelet transform

Wigner-Ville distribution

coefficients of the wavelet transform

time domain signal

average of the signal

shape wavelet parameter

frequency resolution of the wavelet

filter

wavelet function

dilated and translated wavelet

complex conjugate 


\section{INTRODUCTION}

Vibration in a reciprocating internal combustion diesel engine occurs owing to unidirectional combustion forces and structural resonances [1]. Any change in the fuel injection pattern during the ignition delay period affects the quality and the timing of the combustion process, as well as the vibration levels of the engine structure. Especially at low engine idle speeds $(600-800 \mathrm{r} / \mathrm{min})$, fuel economy and reduced emission requirements lead to increased combustion irregularity $[\mathbf{2}, \mathbf{3}]$. Periodic oscillations in engine idle speed are caused by the cyclic combustion process that occurs in each cylinder, resulting in a firing frequency that excites resonant vibrations of vehicle mechanical structures such as the steering system. Typical vibrational modes of steering wheel components (wheel and column assembly) normally lie between 20 and $50 \mathrm{~Hz}[4]$. Superposed on this periodic variation are fluctuations in crankshaft angular velocity that are caused by the stochastic nature of the combustion process from cycle to cycle and by an unequal distribution of fuel from cylinder to cylinder [5]. Typical frequencies seen in vibration power spectra measured on the engine block include low-frequency harmonic components at the firing frequency and its multiples from $1 \mathrm{~Hz}$ up to $400 \mathrm{~Hz}$, bending frequencies in the range 400-800 Hz [1] and combustion chamber resonances in the range $800-4000 \mathrm{~Hz}$ [6]. Engine idle vibration therefore belongs to a class of waveforms characterized by a mixture of deterministic components and non-stationary random components. When cycle-to-cycle variations (CCVs) are large, the driver perceives a rough or unsteady idle vibration, which can be interpreted as poor vehicle performance or poor fuel quality.

Owing to the non-stationary behaviour of the signals affected by the CCVs, classical Fourier analysis is not entirely suitable since it is based on the assumption of stationarity. In Fourier analysis the averaging process can lead to a loss of information regarding the transient events present in the signal and to the smoothing and averaging of time-variant characteristics in the signal statistics. The use of Hilbert transform based envelopes and instantaneous frequencies does not give effective results for multicomponent signals, and bandpass filtering processes are also required [7]. In order to obtain information about the instantaneous character of a signal, time-frequency analysis is often used [8]. Such an analysis gives local information pertaining to the temporal variations in the spectral content of the signal. Some examples of time-frequency methods are the short-time Fourier transform (STFT), the Wigner-Ville distribution (WVD) and wavelet analysis. The wavelet transform (WT) has the advantage of a linear representation and as such does not suffer from interference terms as in the case of the WVD [9]. In contrast to the STFT, in which the time-frequency resolution is uniform, the WT can be interpreted as a filter of constant relative frequency resolution $\Delta f / f[\mathbf{1 0}]$. In this way, the variation in the instantaneous characteristics can be more closely monitored.

The aim of this paper is to compare the effectiveness and sensitivity of different vibration analysis techniques for detecting and quantifying the variation in steering wheel vibration caused by engine idle irregularity. Section 2 outlines each technique and its properties concerning the detection of the non-stationary characteristics of the signals. In addition, normalized indices of variability have been proposed as indicators of engine vibration variation. Section 3 describes the experimental procedure used and the vehicles whose idle vibration was measured. The experimental results of the instantaneous and cycle-to-cycle variation characteristics of the steering wheel vibrations are presented in section 4 . In section 5 the performance of the methods is compared, and section 6 presents a summary of the merits and demerits of the outlined techniques when applied to the analysis of engine idle vibration.

\section{SIGNAL PROCESSING THEORY}

\subsection{Wavelet transform based analysis for diesel idle vibration}

In order to identify both instantaneous and cyclic variations, a quantitative assessment was made by means of:

(a) extraction of the ridge and skeleton of the continuous wavelet transform (CWT) [8];

(b) extraction of periodicities from the discrete orthogonal wavelet transform (OWT) [11] using time-varying autocovariance (TV-AutoCov) analysis [12].

From the array of the instantaneous and cycle-to-cycle characteristics, the time-average value, standard deviation and coefficient of variation $(\mathrm{CV}=\mathrm{SD} /$ mean $)$ were calculated and compared so as to:

(a) determine whether the changes were more due to differences in instantaneous value or to differences from cycle to cycle;

(b) verify which properties changed from one fuel or vehicle to another.

All signal processing algorithms employed were coded in the MATLAB software environment $[13,14]$. Figure 1 summarizes the computations and comparisons performed.

\subsubsection{Continuous wavelet transform: extraction of ridge and skeleton of harmonic components}

The continuous wavelet transform (CWT) [10] is a linear transformation that decomposes an arbitrary signal $x(t)$ into a superposition of elementary functions $\psi_{a, b}(t)$ 


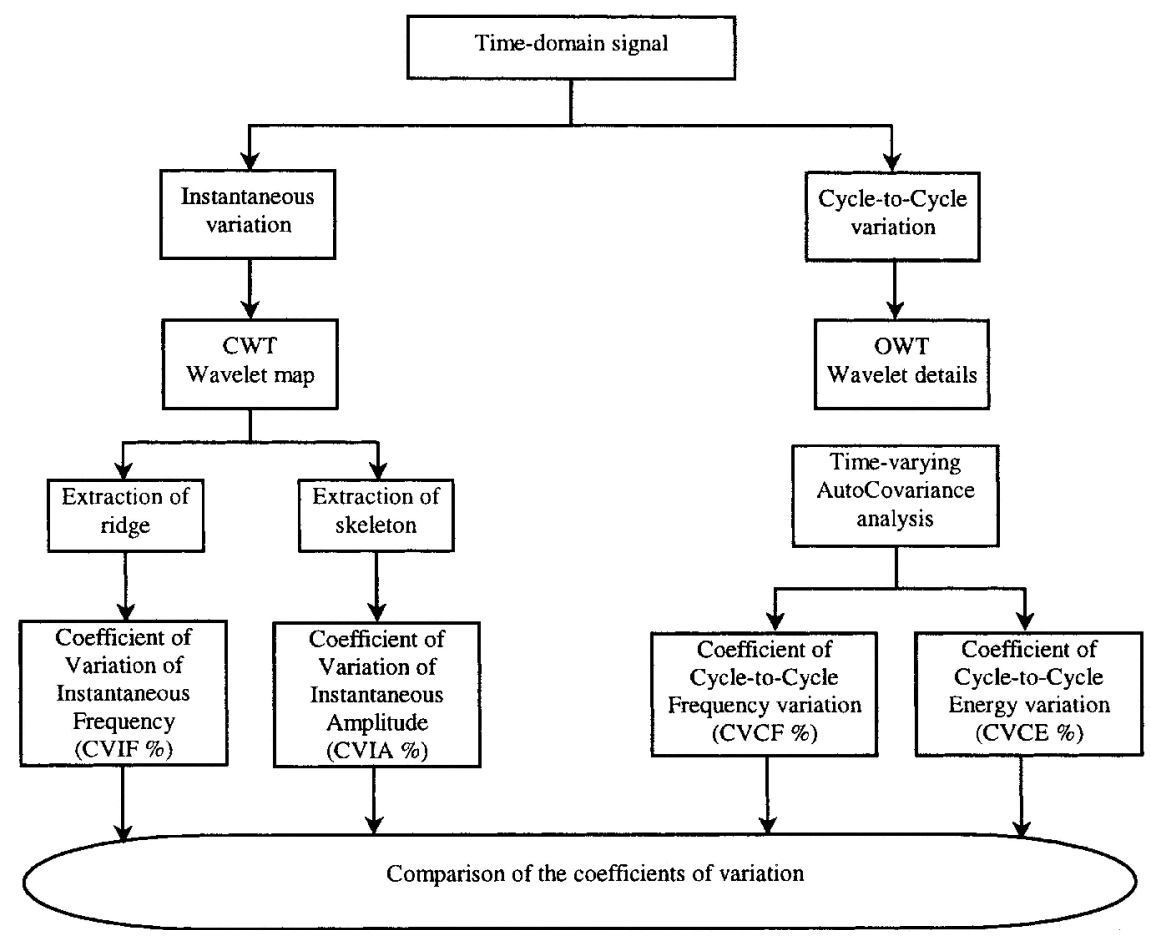

Fig. 1 Flowchart of calculation of coefficients of variation

obtained by a process of scaling and translating a wavelet function $\psi(t)$, called the mother or basic wavelet, i.e.

$$
\psi_{a, b}(t)=\psi *\left(\frac{t-b}{a}\right)
$$

to give the scaled wavelet coefficients $W_{\psi}(a, b)$

$$
W_{\psi}(a, b)=\frac{1}{\sqrt{a}} \int_{-\infty}^{\infty} x(t) \psi *\left(\frac{t-b}{a}\right) \mathrm{d} t
$$

where $*$ denotes complex conjugation, $b$ is a translation parameter indicating the time locality, $a(a>0)$ is a scale parameter and the factor $1 / \sqrt{a}$ is a normalization term to keep constant the total energy of the scaled wavelets at different scales. In contrast to Fourier analysis which has an infinite domain of definition, the wavelet basis functions have finite domains, allowing a better identification of non-stationary characteristics. CWT results are often represented graphically in the time-scale plane. In this work, all wavelet maps have been displayed in the time-frequency domain using the relationship

$$
a=\frac{f_{\mathrm{s}} \cdot f_{0}}{f_{x}}
$$

where $f_{x}$ is the frequency of the signal at which the scaled wavelet is focused, $f_{\mathrm{s}}$ is the sampling frequency of the signal and $f_{0}$ is the frequency of oscillation of the wavelet. For the analysis performed in this paper, the Morlet wavelet function has been chosen as the analysing wavelet. It is defined as the product of a complex exponential wave and a Gaussian envelope

$$
\psi(t)=\mathrm{e}^{2 \pi \mathrm{i} f_{\mathrm{O}} t} \cdot \mathrm{e}^{-\beta^{2} t^{2} / 2}
$$

where $f_{0}$ is the frequency of oscillation inside the modulated envelope, $\beta$ is a parameter that controls the shape of the basic wavelet and $t$ is the time. The values $f_{0}=$ $0.875 \mathrm{~Hz}$ and $\beta=0.6$ were used for all analyses. The square of the modulus of $W_{\psi}(a, b)$ (scalogram) can be interpreted as an energy density distribution [8] over the $(a, b)$ time-frequency plane. The energy of a signal on this plane is mainly concentrated around so-called ridges of the wavelet transform, along which the $W_{\psi}(a, b)$ reach a maximum. The values of the coefficients restricted to the ridges form the skeleton of the wavelet transform.

\subsubsection{Orthogonal wavelet transform: extraction of periodicities from the wavelet coefficients using time-varying autocovariance analysis}

Time-frequency CWT analysis can be confined to a discrete set of translation and scale parameters. Within the dyadic framework, i.e. $a_{j}=2^{j}$ and $b_{j, k}=k / 2^{j}$, the discrete orthogonal wavelet transform (OWT) can be defined. Function $\psi(t)$ is called an orthogonal wavelet if the family

$$
\begin{aligned}
& \psi_{j, k}(t)=2^{j / 2} \psi\left(2^{j} t-k\right), \\
& \text { with } j=1,2, \ldots, J \quad(j, k \in Z)
\end{aligned}
$$

forms an orthonormal basis of functions, where $J$ denotes the number of levels $j$ of the decomposition, and $k$ represents the number of wavelet coefficients. The orthogonal wavelet transform then becomes

$$
x_{k}^{j}=\int_{-\infty}^{\infty} x(t) \psi_{j, k}(t) \mathrm{d} t
$$


In contrast to the CWT, the OWT does not lead to any redundancies in the analysis. A number of wavelet functions satisfy the admissibility conditions for orthogonal analysis. To analyse the engine and steering wheel vibrations, a Daubechies wavelet of the twentieth order (DB20) was used. Each level $j$ of the decomposition consists of convolving the analysed signal with low-pass and high-pass filters and downsampling. The algorithm allows a maximum of $\log _{2} N$ levels of decomposition, where $N$ is the number of data points in the analysed signal. The outputs of the high-pass filter are called the 'detail coefficients' $c_{\mathrm{D}_{j, k}}$, while the outputs of the lowpass filter are called the 'approximation coefficients' $c_{\mathbf{A}_{j, k}}$. Given the sampling frequency $f_{\mathrm{s}}$ at each level $j$, the detail and approximation coefficients lie within a frequency band given by

$$
\begin{array}{ll}
c_{\mathrm{D}_{j, k}} & {\left[2^{-(j+1)} f_{\mathrm{s}}, 2^{-j} f_{\mathrm{s}}\right]} \\
c_{\mathrm{A}_{j, k}} & \left(0,2^{-(j+1)} f_{\mathrm{s}}\right]
\end{array}
$$

The detail coefficients obtained for level $j$ can be recombined to reconstruct the signal components in the specific frequency band [10]. For the current application, the choice of a high decomposition level order $\left(J=\log _{2} N\right)$ made it possible to separate each $K$ th engine harmonic into a separate frequency band. In order to extract the cycle periods, and thus the frequency and amplitude cycle-to-cycle variation of each harmonic, autocorrelation analysis was employed on the wavelet detail level. For a signal $x(t)$, the autocovariance is expressed as

$$
R_{x}(\tau)=E\{[x(t)-\bar{x}] \cdot[x *(t+\tau)-\bar{x}]\}
$$

where $E$ is the expected value operator, * denotes the complex conjugate, $\bar{x}$ is the mean value of $x(t)$, and $\tau$ is the time lag by which the signal is shifted to assess selfsimilarity. In order to examine the changes in periodicity of the engine firing frequency from cycle to cycle, a timevarying autocovariance (TV-AutoCov) method [12] was applied over the selected level detail component using a sliding window of finite length $T$ equal to the average period of a working cycle. The time lag $\tau$ of the first right peak neighbouring the zero lag $(\tau=0)$ provided the cycle period (CP in Fig. 2), whereas the height of the central lobe provided the mean square value of one cycle, a measure of the energy content within a working cycle. By calculating for each window the cycle period $\mathrm{CP}$ and the difference between the height of the central lobe and that of the first right side lobe (indicated in Fig. 2 as delta peak height), it was possible to track the changes in frequency and energy from cycle to cycle.

\subsection{Indices of variability}

For each fuel $m$ and each vehicle tested, the coefficient of variation $\mathrm{CV}_{m}=(\mathrm{SD} /$ mean $) * 100 \%$ was determined for the following parameters: the instantaneous frequency variation (CVIF) found in the continuous wave- let transform, the instantaneous amplitude variation (CVIA) found in the continuous wavelet transform, the cycle-to-cycle frequency variation (CVCF) of the TV-AutoCov analysis and the cycle-to-cycle energy variation (CVCE) of the TV-AutoCov analysis. In order to compare the effectiveness of the two methods under consideration and to compare the behaviour of the two test vehicles, an overall measure of variation was also determined across all the fuels tested. The coefficients of variation for each fuel were averaged to determine the overall index of intrafuel variability $S_{1}$ for both vehicles, defined as

$$
\begin{aligned}
S_{1} \% & =\text { average coefficient of variation across all fuels } \\
& =\left\langle\mathrm{CV}_{m}\right\rangle
\end{aligned}
$$

where the symbol $\langle>$ represents the average taken across all fuels.

\section{EXPERIMENTAL IDLE VIBRATION TESTS}

\subsection{Choice of fuels and vehicles}

Twelve diesel fuels (properties listed in Table 1) were tested under idle conditions in two vehicles. The cetane number of the test fuels ranged from 27.1 to 77.0 in order to represent fuels having different combustion characteristics. Table 2 lists the two vehicles tested and their basic engine specifications.

In order to introduce variation into the data, an older vehicle with a prechamber-based indirect combustion system was chosen along with a vehicle having a modern direct injection common rail system. After each change of fuel, each vehicle was left to idle for approximately $10 \mathrm{~min}$ so as to stabilize temperature and injection conditions.

\subsection{Measurements and operating conditions}

Vibration measurements were made at two points on each vehicle:

(a) on the upper part of the cylinder blocks,

(b) on the surface of the steering wheel at the two o'clock position.

The directions of measurement at the engine block were taken along the three principal axes of the car (see Fig. 3). The axial direction $Y$ was taken along the crankshaft axis, the vertical direction $Z$ was taken upwards, while the transverse direction $X$ was in the horizontal plane normal to the crankshaft axis. The directions of measurement at the steering wheel were chosen following the guidelines provided by British Standard 6842 [15]:

1. $\left(y^{\prime}, z^{\prime}\right)$ defines the plane of the steering wheel, where the $y^{\prime}$ axis (lateral direction) is directed towards the left of the vehicle, the $z^{\prime}$ axis (vertical direction) is 


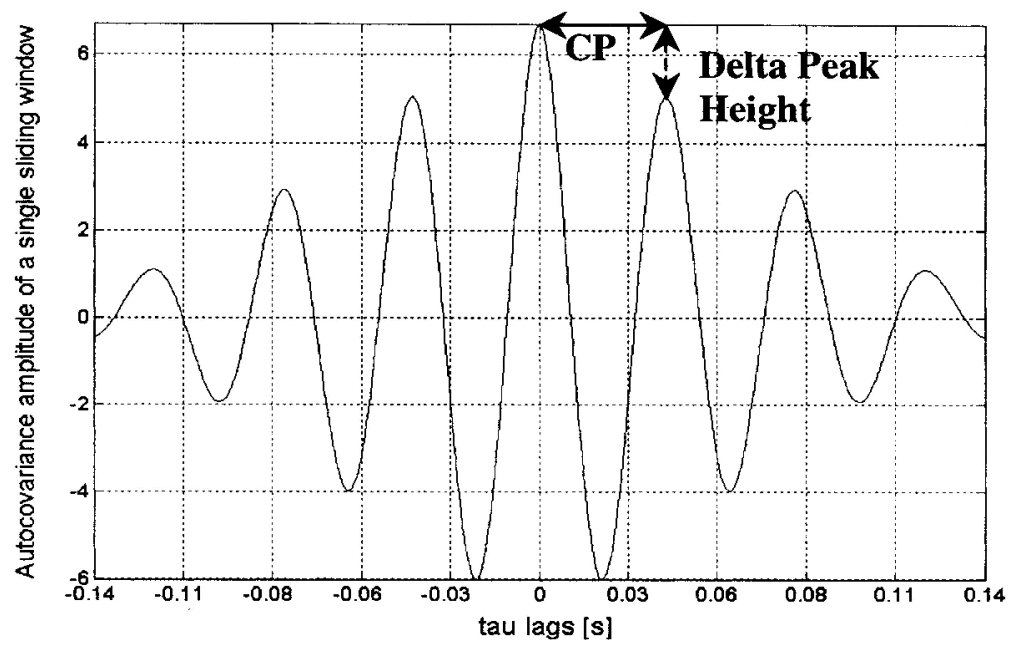

Fig. 2 Autocovariance sequence obtained over one time window. CP indicates the cycle period

Table 1 Test diesel fuel properties

\begin{tabular}{clll}
\hline Fuel & Density $\left(\mathrm{kg} / \mathrm{m}^{3}\right)^{*}$ & Viscosity $\left(\mathrm{mm}^{2} / \mathrm{s}\right)^{\dagger}$ & Cetane number \\
\hline 1 & 773.1 & - & 77.0 \\
2 & 824.1 & 2.27 & 52.9 \\
3 & 826.9 & 3.19 & 63.3 \\
4 & 811.4 & 2.94 & 69.3 \\
5 & 837.3 & 2.93 & 52.4 \\
6 & 873.3 & 3.40 & 50.7 \\
7 & 830.1 & 2.72 & 54.5 \\
8 & 817.7 & 1.95 & 54.9 \\
9 & 837.6 & 3.33 & 56.5 \\
10 & 847.4 & 2.22 & 44.7 \\
11 & 836.1 & 1.93 & 41.4 \\
12 & 915.3 & - & 27.1 \\
\hline
\end{tabular}

* Density at $15^{\circ} \mathrm{C}$.

${ }^{\dagger}$ Kinematic viscosity at $40^{\circ} \mathrm{C}$.

the tangent of the steering wheel directed towards the windscreen.

2. The $x^{\prime}$ axis (fore-aft direction) is normal to plane $y^{\prime}, z^{\prime}$.

The engine block and steering wheel vibrations were measured by means of triaxial piezoresistive accelerometers (Entran EGAS3-CM-25). The accelerometers were fixed at the measurement locations by means of aluminium blocks and mounting screws. The acceleration signals were amplified by an Entran MSC6 signal-

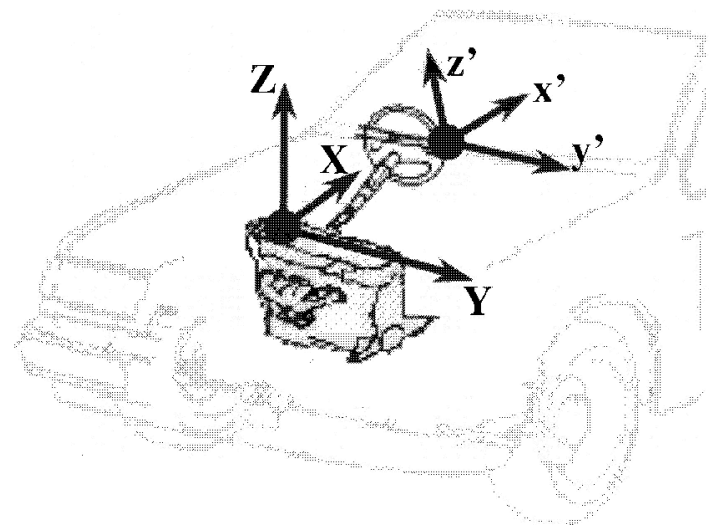

Fig. 3 Directions of vibration measurements for the engine block and steering wheel

conditioning unit [16], stored using six channels of a digital audio tape (DAT) recorder (PC216A SONY) [17] and monitored by a Tektronix TDS210 digital oscilloscope [18]. The amplifier and the DAT recorder were run using a d.c. battery to eliminate electronic noise from the vehicle systems. The sampling frequency of the DAT unit was $5 \mathrm{kHz}$. The recorded signals were reacquired and analysed in the laboratory by means of a Hewlett Packard 9000 C110 Unix workstation running the Fourier Monitor module of the LMS CADA-X 3.5B

Table 2 Diesel vehicles tested

\begin{tabular}{lll}
\hline & \multicolumn{2}{c}{ Vehicle model } \\
\cline { 2 - 3 } & Renault 19 & Ford Focus \\
\hline Specification & $\begin{array}{l}\text { 1.91 turbocharged diesel, indirect } \\
\text { injection (IDI), precombustion }\end{array}$ & $\begin{array}{c}1.81 \text { turbocharged diesel, direct } \\
\text { injection (DI), common rail fuel }\end{array}$ \\
& $\quad$ chamber system & injection system \\
Engine type & Four-cylinder, dual overhead cam & Four-cylinder, dual overhead cam \\
Bore $(\mathrm{mm})$ & 80 & 82.50 \\
Stroke $(\mathrm{mm})$ & 93 & 82 \\
Displacement $\left(\mathrm{cm}^{3}\right)$ & 1870 & 1753 \\
Compression ratio & $21.5 / 1$ & $19.40 / 1$ \\
\hline
\end{tabular}


software [19], using a 12-channel Difa System Scadas front-end unit. Since the ultimate objective of the investigation was to identify the characteristics and variation in idle vibration signals in preparation for future studies investigating the subjective reaction to idle vibration, the signals were sampled at $2048 \mathrm{~Hz}$, a rate that is adequate for that purpose.

The vibration signals were measured for both a Renault 19 and a Ford Focus 1.8 in the following test conditions:

(a) engine standing at idle;

(b) transmission in neutral;

(c) no human subject sitting in the car during the test or holding the steering wheel;

(d) 12 diesel fuels having different cetane numbers;

(e) data recorded over 2 min for each fuel;

(f) $20^{\circ} \mathrm{C}$ environmental temperature and 40 per cent humidity.

\section{ANALYSIS OF THE RESULTS}

The operation conditions and some basic constants for a four-cylinder engine are shown in Table 3 in order to understand the frequencies observed on the steering wheel signals. They are described as follows:

$$
\begin{aligned}
& n=\text { nominal idling speed of crankshaft }(\mathrm{r} / \mathrm{min}) \\
& T_{\mathrm{REV}}=\frac{(60 \mathrm{~s} / \mathrm{min})}{(n \mathrm{r} / \mathrm{min})}=\text { period of one revolution of shaft } \\
& T_{\mathrm{C}}=2 T_{\mathrm{REV}}=\text { period of one working cycle } \\
& F_{\mathrm{REV}}=\frac{1}{T_{\mathrm{REV}}}=\text { revolution frequency of crankshaft } \\
& F_{\mathrm{C}}=1 / T_{\mathrm{C}}=\text { working cycle repetition frequency } \\
& F_{\mathrm{F}}=\frac{(\text { cylinders }) *(n \mathrm{r} / \mathrm{min})}{(2 \mathrm{r} / \mathrm{cycle}) *(60 \mathrm{~s} / \mathrm{min})}=\text { firing frequency } \\
& T_{\mathrm{F}}=\frac{1}{F_{\mathrm{F}}}=\text { period between consecutive cylinder firings }
\end{aligned}
$$

Figures $4 \mathrm{a}$ and $\mathrm{c}$ present typical accelerometer traces recorded from the engine block, while Figs $4 \mathrm{~b}$ and $\mathrm{d}$ present the same for the steering wheel for fuel 9 used in Renault 19 and Ford Focus respectively. The time histories are $1 \mathrm{~s}$ in length, which corresponds to approximately 28 individual cylinder combustions. The analysis of the results is shown for the $X$ axis measured at the engine block, since this was the direction with the highest vibration level. The vibration energy along the vertical ( $Z$ axis) direction presented similar features but with lower intensity, whereas that experienced along the axial ( $Y$ axis) direction was very low and was found not to have a significant influence on the steering wheel energy levels. As far as the steering wheel vibration levels are concerned, the vertical axis $z^{\prime}$ along the tangent of the steering wheel was chosen for the analysis since the energy level was higher in this direction.

As shown in Fig. 4, fluctuations in the periodic activity of the combustion process lead to changes in combustion duration $T_{\mathrm{F}}$ (time between consecutive cylinder firings), to amplitude modulation of the combustion activity from cycle to cycle and to an unequal combustion peak distribution. In addition, the two vehicles differed in the energy transmitted to the steering wheel, resulting in more attenuation for the Ford Focus than for the Renault 19 but with greater modulation in frequency. Fourier analysis of the engine block and steering wheel signals of Fig. 4 are presented in Fig. 5 in power spectral density form along with the associated transfer and coherence functions. The acceleration transmissibility was estimated using the $\mathrm{Hv}$ spectral estimator which assumes noise on both input and output signals. All calculations were performed using a block size of 2048 points and an overlap of 75 per cent, leading to a spectral resolution of $1.0 \mathrm{~Hz}$.

The spectra of the engine block shown in Figs 5a and $\mathrm{a}^{\prime}$ exhibit a double peak valley structure: low-frequency components (up to $400 \mathrm{~Hz}$ ) at the firing frequency and its multiples and high-frequency broad peaks that were not related to engine speed but were due mainly to bending vibrations of the engine structure $(400-800 \mathrm{~Hz})$. The power spectral densities of the steering wheel acceleration signals shown in Figs $5 \mathrm{~d}$ and $\mathrm{d}^{\prime}$ show that the energy conveyed to the steering wheel covered only the low-frequency range up to $200 \mathrm{~Hz}$, and that it was mostly due to even-order engine harmonics $\mathrm{H}_{2}, \mathrm{H}_{4}$ and $\mathrm{H}_{6}$ transmitted through the moving parts of the engine, whereas the major part of the vibration transmission from the combustion process was attenuated in the path through the steering system, as shown by the transmissibility function in Figs $5 \mathrm{c}$ and $\mathrm{c}^{\prime}$. The operating cycle of the engine introduces time-varying forces that make the harmonic orders non-stationary in frequency when combustion takes place. Different fuel content can affect this variability significantly. However, FFT analysis does not identify this process since it only gives global energy information about the frequency content.

Table 3 Basic constants for the test vehicles when at idle

\begin{tabular}{lllllllll}
\hline Type of vehicle & $\begin{array}{l}\text { Number of } \\
\text { cylinders }\end{array}$ & $\begin{array}{l}n \\
\mathrm{r} / \mathrm{min}\end{array}$ & $\begin{array}{l}T_{\mathrm{REV}} \\
(\mathrm{s})\end{array}$ & $\begin{array}{l}T_{\mathrm{C}} \\
(\mathrm{s})\end{array}$ & $\begin{array}{l}F_{\mathrm{REV}} \\
(\mathrm{Hz})\end{array}$ & $\begin{array}{l}F_{\mathrm{C}} \\
(\mathrm{Hz})\end{array}$ & $\begin{array}{l}F_{\mathrm{F}} \\
(\mathrm{Hz})\end{array}$ & $\begin{array}{l}T_{\mathrm{F}} \\
(\mathrm{s})\end{array}$ \\
\hline Renault 19 & 4 & 840 & 0.071 & 0.143 & 14 & 7 & 28 & 0.036 \\
Ford Focus 1.8 & 4 & 780 & 0.077 & 0.154 & 13 & 6.5 & 26 & 0.038 \\
\hline
\end{tabular}




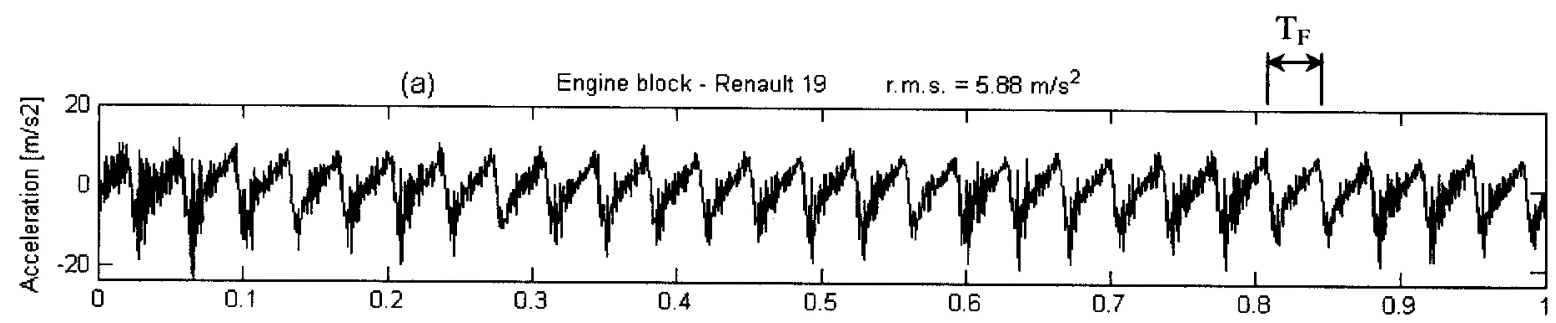

(b)

Steering wheel - Renault $19 \quad \mathrm{r} . \mathrm{m} . \mathrm{s} .=3.05 \mathrm{~m} / \mathrm{s}^{2}$

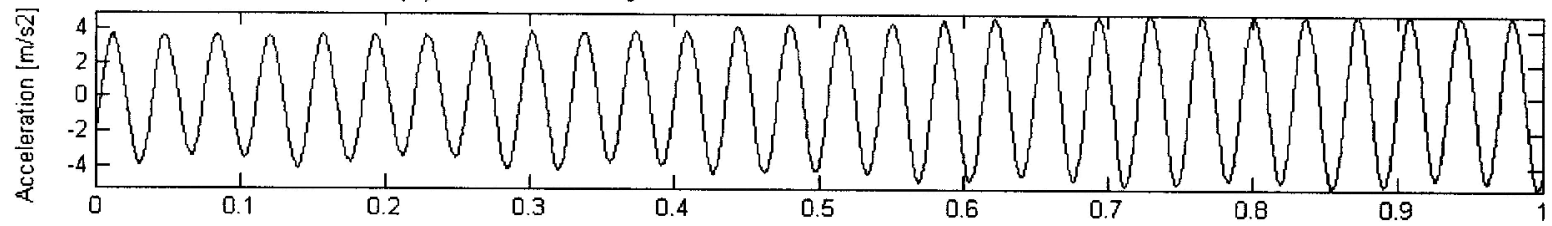

(c) Engine block - Ford Focus r.m.s. $=5.08 \mathrm{~m} / \mathrm{s}^{2}$

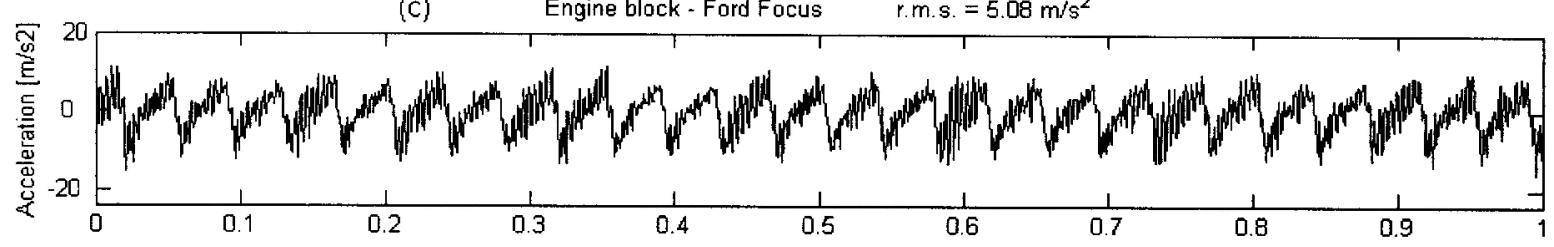

(d) Steering wheel - Fard Focus r.m.s. $=0.56 \mathrm{~m} / \mathrm{s}^{2}$

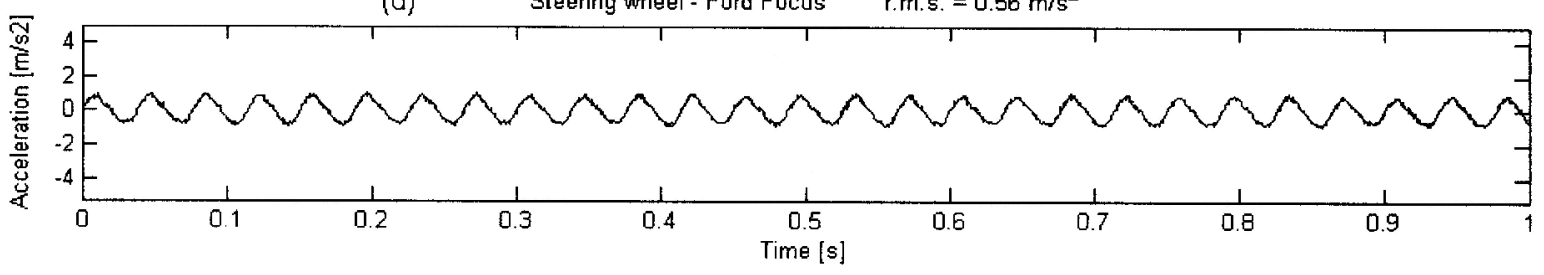

Fig. 4 Idle acceleration time histories recorded for fuel 9: (a) engine block vibration along $X$ axis for Renault 19; (b) steering wheel vibration along $z^{\prime}$ axis for Renault 19; (c) engine block vibration along $X$ axis for Ford Focus; (d) steering wheel vibration along $z^{\prime}$ axis for Ford Focus

\subsection{Application of the continuous wavelet transform}

Figure 6 presents in the form of a greyscale intensity plot an example of CWT analysis as applied to the engine block acceleration of the Renault 19. Figure 6b suggests that most of the energy in the vibration signal was centred at a firing frequency of $28 \mathrm{~Hz}$ and at even-order harmonics of $F_{\mathrm{REV}}(14 \mathrm{~Hz})$, represented by horizontal dark bands in the low-frequency range $(0-400 \mathrm{~Hz})$ along the time axis, whereas the high-frequency range $(400-1024 \mathrm{~Hz})$ is characterized by an impulsive nature of the combustion process, represented by a more discrete pattern showing transitions from white (low energy) to black (high energy), indicating the periodicity of the firing process.

In order to examine the sensitivity of the method to changes in the instantaneous characteristics of the steering wheel vibration, the ridge and skeleton of the CWT of harmonics $\mathrm{H}_{2}$ and $\mathrm{H}_{4}$ were obtained. It was decided to investigate these two harmonic orders since they were more prominent. Figures $7 b$ to $e$ and $b^{\prime}$ to $\mathrm{e}^{\prime}$ present the scalogram of the CWT corresponding to 55 engine cycles ( $8 \mathrm{~s}$ of steering wheel time history) for fuel 11 used for the Renault 19 and Ford Focus respectively. Here, the variation in the firing frequency resulted in an oscillating band along the time axis, whereas the amplitude modulation took the form of an alternating intensity from white to black areas. The plots in Figs 7c to $f$ and $c^{\prime}$ to $f^{\prime}$ show the instantaneous frequency characteristics obtained from the wavelet ridge, while Figs $7 \mathrm{~d}$ to $\mathrm{g}$ and $\mathrm{d}^{\prime}$ to $\mathrm{g}$ ' show the instantaneous envelope (local maxima amplitude variation) characteristics of the analysed harmonic obtained from the wavelet skeleton.

The coefficients of variation describing the changes in ridge frequency and those describing the changes in amplitude for all fuels, sorted in order of decreasing cetane number, are presented in Fig. 8. It can be noted that the variations in the amplitude of the signal harmonics were found to be higher than the variations in the frequency. The figure also suggests that for the Renault 19 the variations in harmonic $H_{2}$ for fuel 11 and fuel 12 are higher than those for all the other fuels. This feature can be attributed to the very low cetane numbers involved. In particular, fuel 12 has a $\mathrm{CN}$ of 27.1 , that is, a value out of the standard specification 

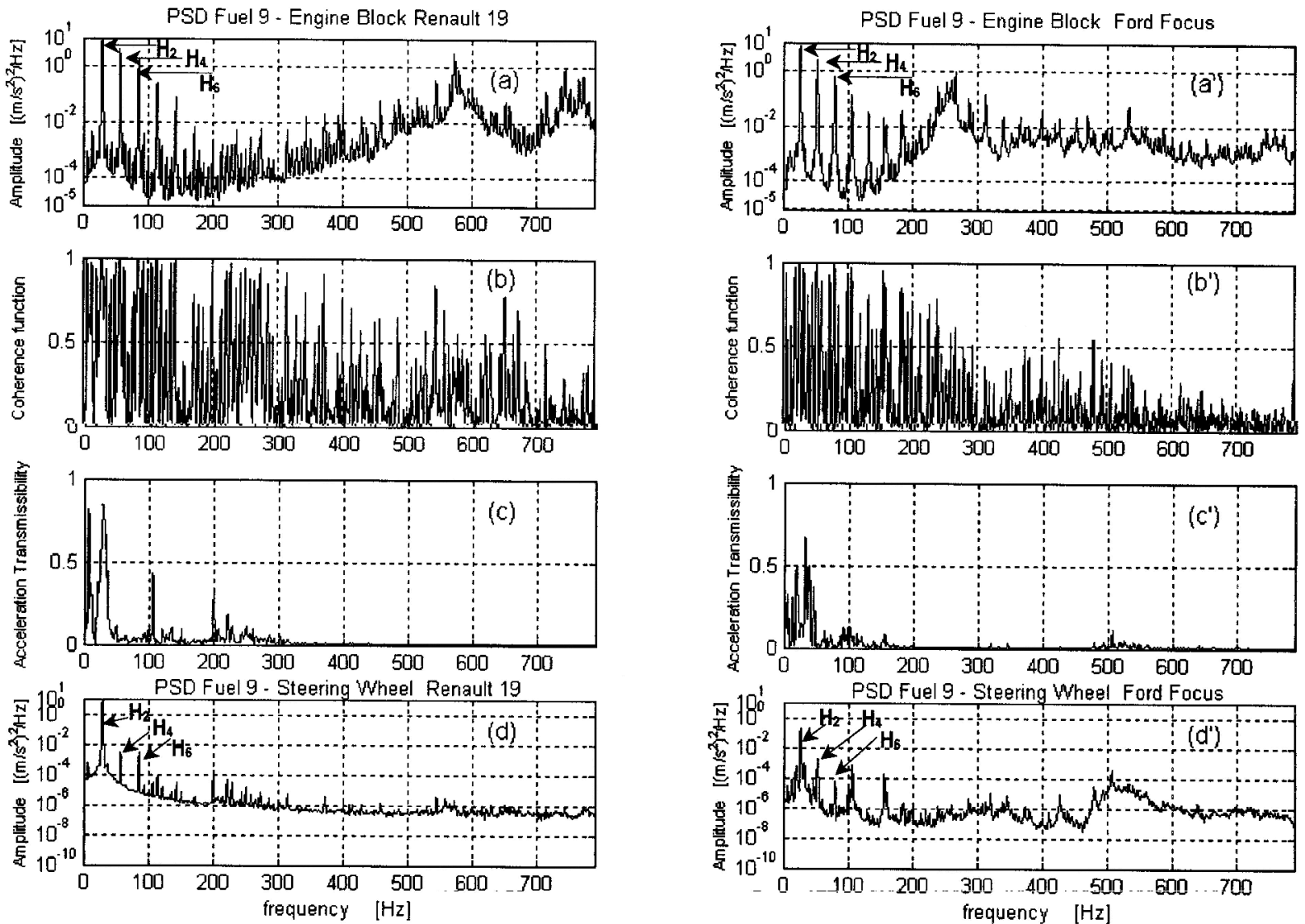

Fig. 5 (a, a') Acceleration PSD engine block, $\left(b, b^{\prime}\right)$ coherence function, (c, $\left.c^{\prime}\right)$ transfer function and $\left(d, d^{\prime}\right)$ acceleration PSD steering wheel vibration for Renault 19 and Ford Focus respectively. Frequency resolution $1 \mathrm{~Hz}$. Sampling frequency $2048 \mathrm{~Hz}$. Sample duration 2 min
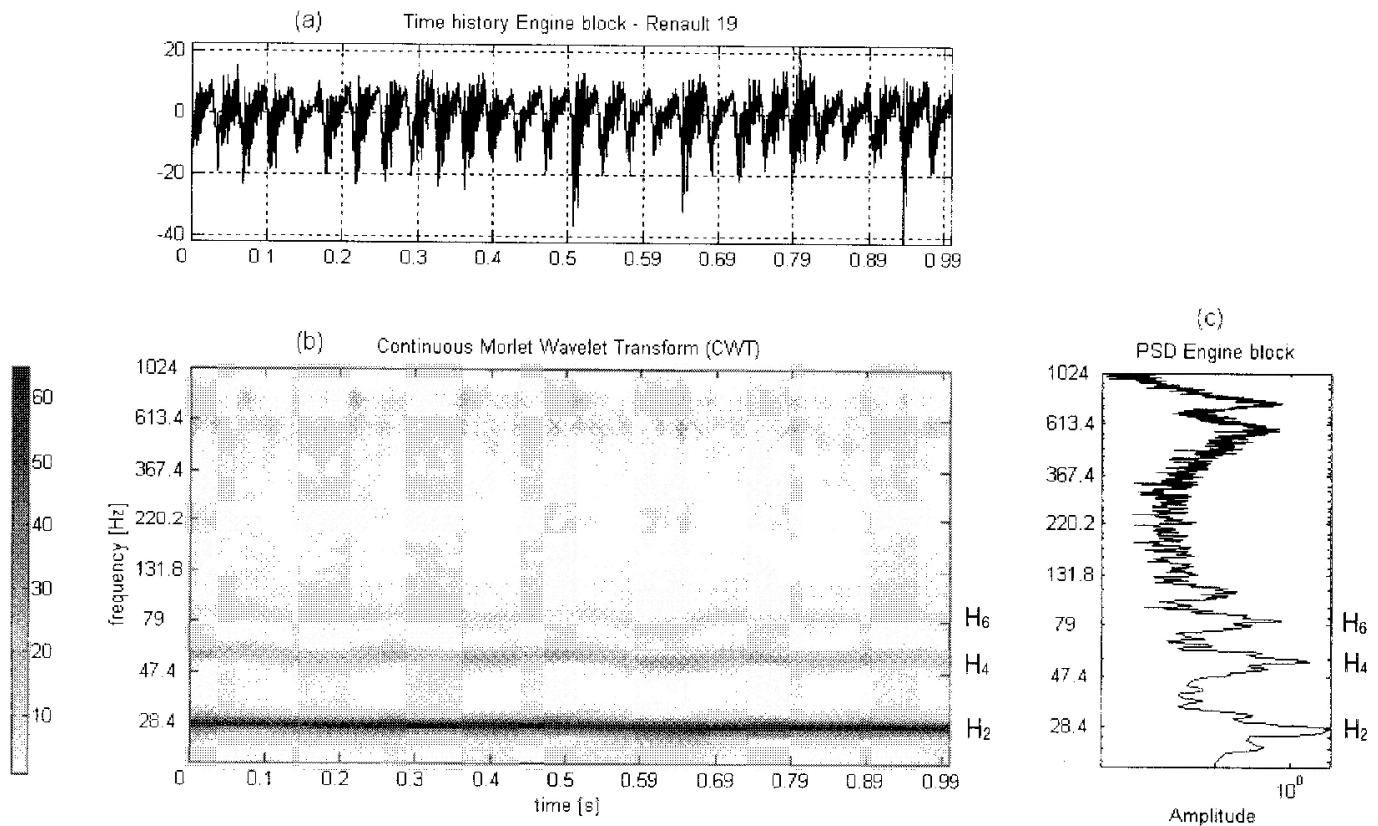

Fig. 6 Engine block vibration: (a) time history; (b) modulus of continuous wavelet transform; (c) PSD 

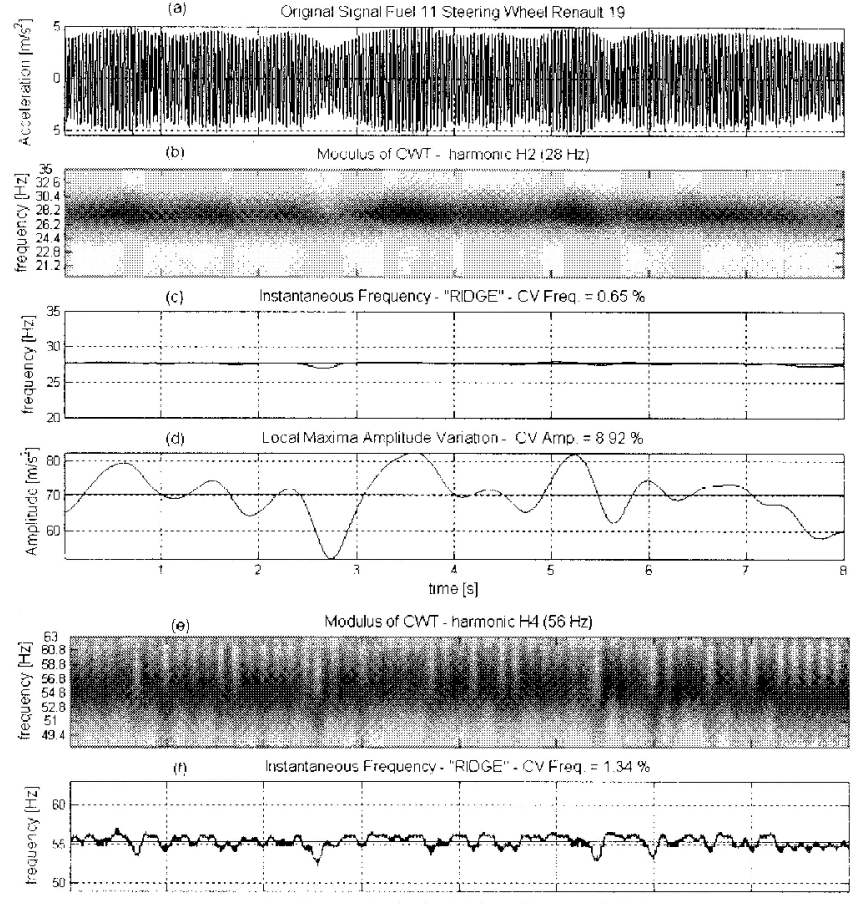

(9)

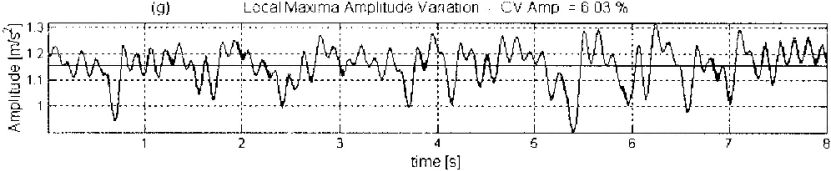

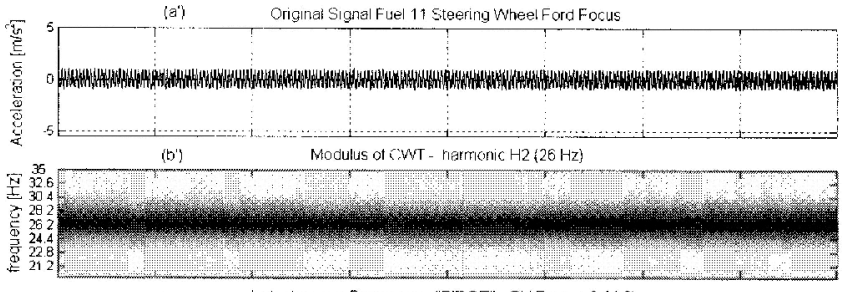
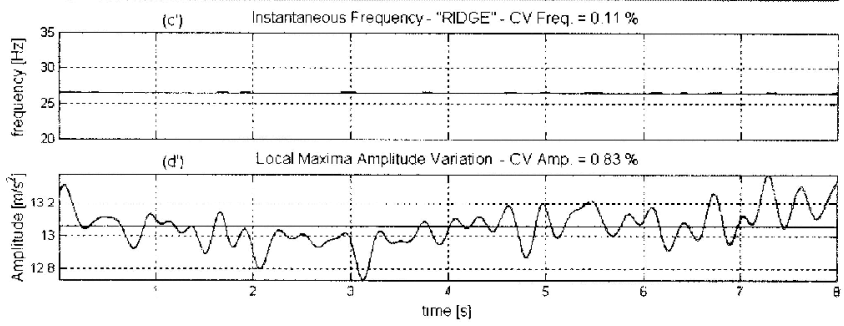

(e')

Modulus of $\mathrm{CWT}$. harmonic $\mathrm{H} 4\{52 \mathrm{~Hz})$
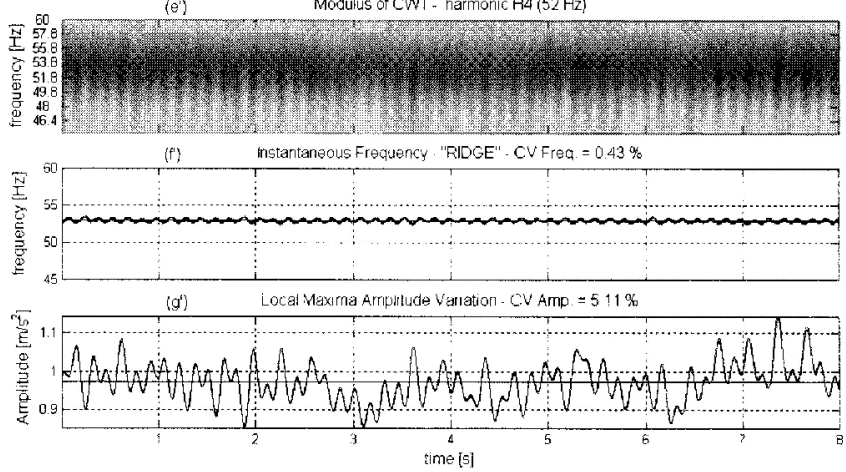

Fig. 7 Ridge and envelope extracted from the CWT of steering wheel acceleration of the Renault 19 and Ford Focus: $\left(a, a^{\prime}\right)$ original signal; ( $\left.b, b^{\prime}\right)$ modulus of CWT on harmonic $H_{2} ;\left(c, c^{\prime}\right)$ instantaneous frequency on harmonic $H_{2} ;\left(\mathrm{d}, \mathrm{d}^{\prime}\right)$ instantaneous envelope on harmonic $H_{2} ;\left(\mathrm{e}, \mathrm{e}^{\prime}\right)$ modulus of CWT on harmonic $H_{4} ;\left(\mathrm{f}, \mathrm{f}^{\prime}\right)$ instantaneous frequency on harmonic $H_{4} ;\left(\mathrm{g}, \mathrm{g}^{\prime}\right)$ instantaneous envelope on harmonic $\mathrm{H}_{4}$

for diesel fuel oils $[\mathbf{2 0}]$ where typical ranges of $\mathrm{CN}$ are from 40 to a maximum value of 59 .

\subsection{Application of the orthogonal wavelet transform using the time-varying autocovariance method}

Several levels (frequency bands) of the orthogonal wavelet decomposition were used to extract the changes in frequency and amplitude of the harmonics of the steering wheel acceleration as presented in section 2.1.2. In order to reconstruct the signal within a given frequency band, a decomposition of 14 levels was chosen to be the minimum number needed to separate the first even-order engine harmonics, $\mathrm{H}_{2}$ and $\mathrm{H}_{4}$, in different levels. Figure 9 shows an example of the application of TV-AutoCov on detail level 6 obtained for fuel 11 for the Renault 19 (Fig. 9a). In particular, from level 6 containing the second harmonic $H_{2}$ (i.e. the firing frequency $F_{\mathrm{F}}$ ) of the signal, it was possible to track the evolution of the combustion period and the amplitude variation over time. In fact, any engine harmonic could be analysed independently by means of an appropriate choice of wavelet level. Figure $9 \mathrm{~b}$ presents the three-dimensional plot of the windowed autocovariance sequences in the time lag-covariance space.

As shown in Fig. 9c, the changes in the firing frequency over time were indicated in the evolution of the combustion cycle periods, calculated as the time lag of the first dominant side lobe of the autocovariance in each window. As shown in Fig. 9d, the amplitude variations had the same general trend identified by the skeleton of the CWT (see Fig. 7d), but a higher coefficient of variation ( 32.96 per cent as opposed to 8.92 per cent) is found when using TV-AutoCov analysis. This trend was found for all the fuels and for both vehicles tested, as shown in Fig 10.

\section{COMPARISON OF SIGNAL PROCESSING METHOD PERFORMANCE}

Table 4 presents an overall comparison between the CWT analysis and the TV-AutoCov method in terms of the index of intrafuel variability $S_{1}$ (per cent) calculated 

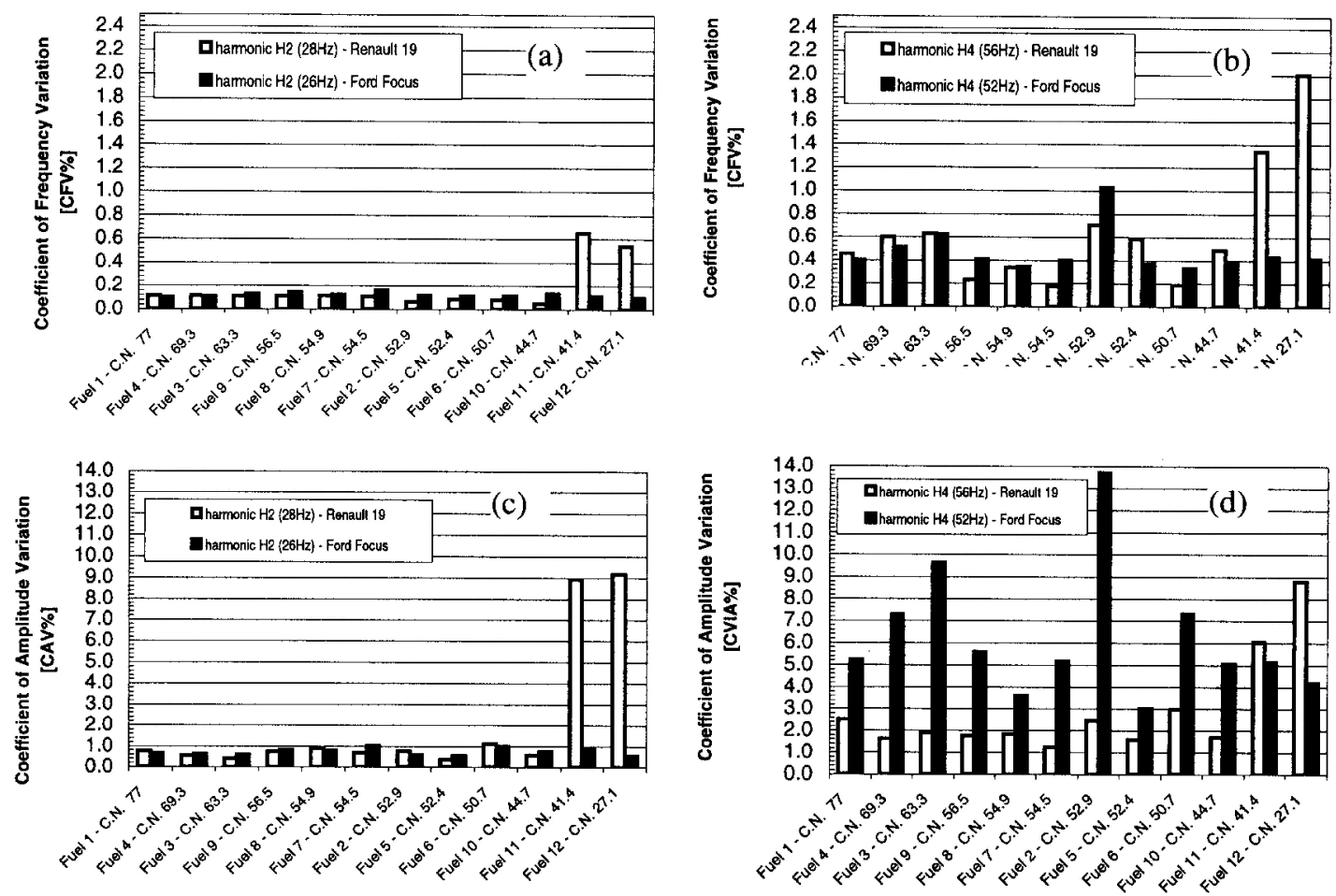

Fig. 8 (a, b) Coefficient of variation in instantaneous frequency (CVIF) and (c, d) coefficient of variation in amplitude (CVIA) on harmonics $\mathrm{H}_{2}$ and $\mathrm{H}_{4}$

for the $\mathrm{H}_{2}$ and $\mathrm{H}_{4}$ harmonics for both vehicles. The values of $S_{1}$ were calculated excluding the results for diesel fuels 11 and 12 since their properties were not representative of any real automotive products.

The TV-AutoCov method was found to be more sensitive to the presence of irregularity and variation within each fuel (higher $S_{1}$ values) around the firing frequency $\left(H_{2}\right)$ and its multiple $\left(H_{4}\right)$ than the CWT ridge and skeleton extraction method. This higher effectiveness was due to the fact that the TV-AutoCov method was computed as a running average on a cycle-to-cycle basis, offering an efficient representation of the changes in the combustion energy from one engine cycle to another. In fact, the ridge and skeleton of the continuous wavelet transform are more suitable for detecting the changes in the instantaneous characteristics of the signal. It can be shown that the CWT produces an exact estimate of the amplitude and frequency modulation law only for asymptotic signals (i.e. signals that have slowly varying amplitude compared with phase variations). When this condition is not met, there exists a bias of the estimation, depending upon the choice of the wavelet function.

The higher intrafuel variability $S_{1}$ values obtained for the amplitude variation using the TV-AutoCov method (a mean value of $S_{1}$ of 22.61 per cent taken across both vehicles) suggested that the variation in the steering wheel vibration was mostly driven by the cycle-to-cycle combustion energy rather than by an unequal instantaneous distribution of combustion peaks as found by the CWT method (2.47 per cent). Also, both methods provide an indication of the nature of diesel idle vibration by showing that the vibration data are more affected by amplitude modulation (a mean value of $S_{1}$ of 12.54 per cent between the two methods) than frequency modulation (4.46 per cent) for all the fuels and vehicles tested.

\section{CONCLUSIONS AND FUTURE DEVELOPMENTS}

The performance and the sensitivity of two signal processing techniques for the detection and quantification of diesel engine idle variation in terms of instantaneous and cyclic irregularity have been assessed. The effectiveness of time-frequency analysis based on the continuous wavelet transform was compared with that of timevarying autocovariance analysis based on the orthogonal wavelet transform when applied to idle steering wheel vibration. The TV-AutoCov analysis was found to be superior to the ridge and skeleton extraction from the continuous wavelet transform when identifying changes in diesel engine idle vibration.

The TV-AutoCov analysis revealed that the steering wheel vibration variation was caused more by the 
(a)

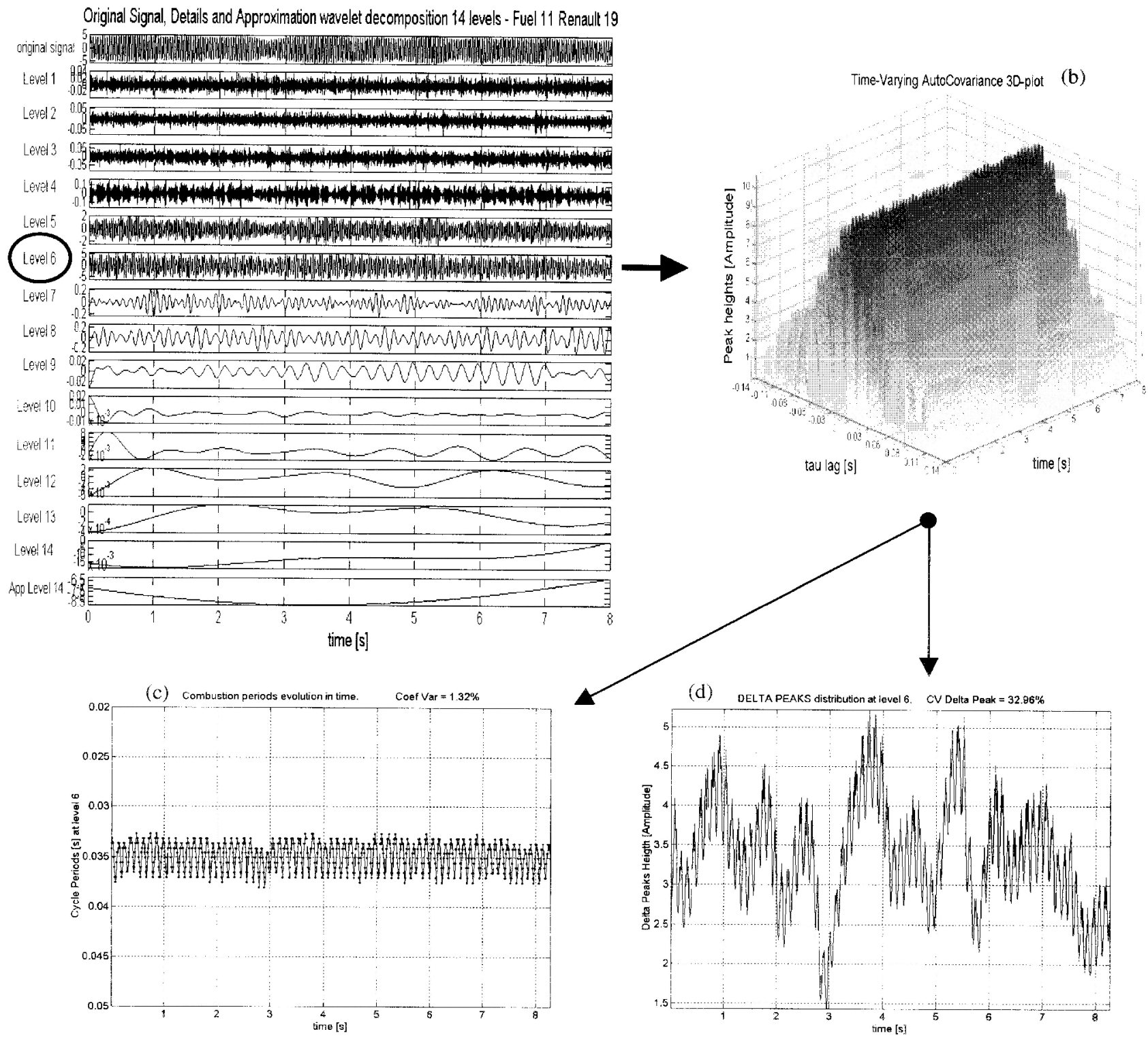

Fig. 9 Time-varying autocovariance method applied to wavelet detail 6 for fuel 11 used on Renault 19: (a) orthogonal wavelet decomposition; (b) three-dimensional plot of windowed AutoCov sequences; (c) changes in combustion periods extracted from TV-AutoCov; (d) changes in combustion energy

cycle-to-cycle combustion energy variation (22.61 per cent) than by an unequal instantaneous distribution of combustion peaks evaluated with the CWT method (2.47 per cent). Both methods showed that diesel idle vibration was more affected by amplitude modulation (12.54 per cent) than frequency modulation ( 4.46 per cent) for all the fuels and for both vehicles tested. Future research will use the methods described here as the basis for defining a set of signals for human subjective response testing with the objective of defining the growth functions of human perceived disturbance as a function of idle vibration amplitude and frequency modulation.

\section{ACKNOWLEDGEMENTS}

The authors would like to express their gratitude to Dr Gautam Kalghatgi and to Dr Wieslaw Staszewski for their many valuable scientific inputs to the research. Thanks are also due to Shell Global Solution UK for their sponsorship and support. 

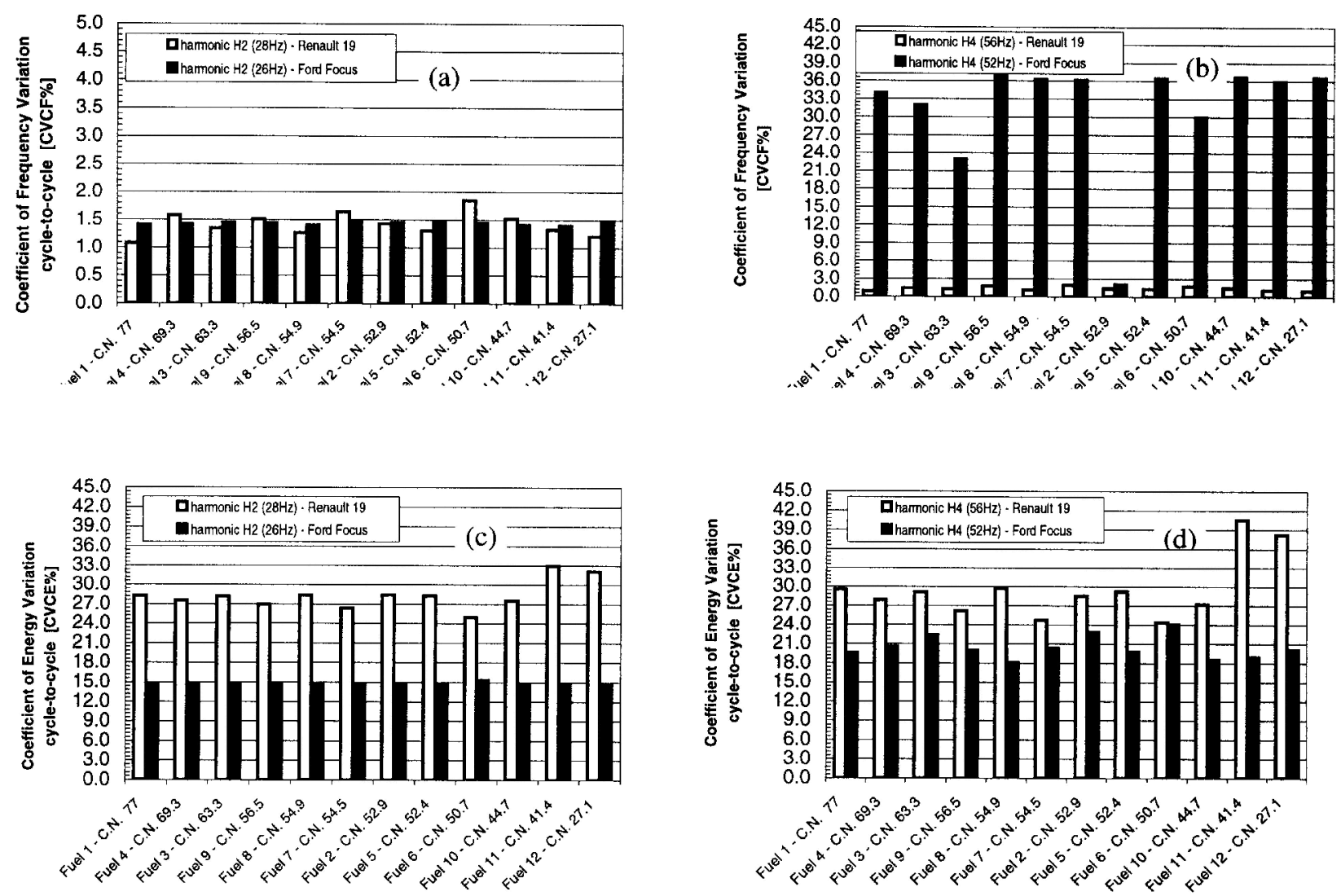

Fig. 10 (a, b) Coefficient of variation in cycle-to-cycle frequency (CVCF) and (c, d) coefficient of variation in energy (CVCE) on harmonics $\mathrm{H}_{2}$ and $\mathrm{H}_{4}$

Table 4 Global intrafuel variability index $S_{1}$ determined across fuels 1 to 10 for harmonics $H_{2}$ and $H_{4}$ of the Renault 19 and Ford Focus test vehicles

\begin{tabular}{|c|c|c|c|c|c|c|}
\hline & \multirow[b]{3}{*}{ Method of analysis } & \multicolumn{4}{|c|}{ Index of intrafuel variability $S_{1}$ (per cent) } & \multirow{3}{*}{$\begin{array}{l}\text { Mean value of } S_{1} \\
\text { (per cent) }\end{array}$} \\
\hline & & \multicolumn{2}{|c|}{ Harmonic $\mathrm{H}_{2}$} & \multicolumn{2}{|c|}{ Harmonic $\mathrm{H}_{4}$} & \\
\hline & & Renault 19 & Ford Focus & Renault 19 & Ford Focus & \\
\hline $\begin{array}{l}\text { Frequency } \\
\text { variation }\end{array}$ & $\begin{array}{l}\text { TV-AutoCov } \\
\text { CWT }\end{array}$ & $\begin{array}{l}1.45 \\
0.10\end{array}$ & $\begin{array}{l}1.43 \\
0.12\end{array}$ & $\begin{array}{l}1.42 \\
0.44\end{array}$ & $\begin{array}{r}30.29 \\
0.48\end{array}$ & $\begin{array}{l}8.64 \\
0.28 \\
\mathbf{4 . 4 6}\end{array}$ \\
\hline $\begin{array}{l}\text { Amplitude } \\
\text { variation }\end{array}$ & $\begin{array}{l}\text { TV-AutoCov } \\
\text { CWT }\end{array}$ & $\begin{array}{r}27.45 \\
0.68\end{array}$ & $\begin{array}{r}14.78 \\
0.73\end{array}$ & $\begin{array}{r}27.63 \\
1.95\end{array}$ & $\begin{array}{r}20.61 \\
6.55\end{array}$ & $\begin{array}{r}22.61 \\
2.47 \\
\mathbf{1 2 . 5 4}\end{array}$ \\
\hline
\end{tabular}

\section{REFERENCES}

1 Priede, T. Noise and vibration control of the internal combustion reciprocating engine. In Noise and Vibration Control Engineering: Principles and Applications (Eds L. L. Bernarek and I. L. Ver), 1992 (John Wiley \& Sons, New York).

2 Ozdor, N., Dulger, M. and Sher, E. Cyclic variability in spark ignition engines: A literaturesurvey. SAE paper 940987, 1994, pp. 1514-1552.

3 Kidoguchi, Y. and Yang, C. Effects of fuel cetane number and aromatics on combustion process and emissions of a direct-injection diesel engine. JSAE Rev., 2000, 21, $469-475$.
4 Pottinger, M. G. and Marshall, K. D. A review of tire/ pavement interaction induced noise and vibration. In The Tire Pavement Interface (Eds M. G. Pottinger and T. J. Yager), 1986, ASTP STP 929 (American Society for Testing and Materials, Philadelphia).

5 Ando, H. and Motomochi, M. Contribution of fuel transport lag and statistical perturbation in combustion to oscillation of SI engine speed at idle. SAE paper 870545, 1987.

6 Ren, Y., Randall, R. B. and Milton, B. E. Influence of the resonant frequency on the control of knock in diesel engines. Proc. Instn Mech. Engrs, Part D: J. Automobile Engineering, 1999, 213, 127-133.

7 Feldman, M. Non-linear system vibration analysis using Hilbert Transform-II. Forced vibration analysis method 
'FORCEVIB'. Mech. Syst. and Signal Processing, 1994, 8, 309-318.

8 Staszewski, W. J. Identification of nonlinear system using multiscale ridges and skeletons of the wavelet transform. J. Sound. Vibr., 1998, 214, 639-658.

9 Feldman, M. Identification of non-linear system parameters via instantaneous frequency: application of the Hilbert transform and Wigner-Ville techniques. In Proceedings of 13th IMAC, Nashville, Tennessee, 1995, pp. 637-642.

10 Mallat, S. A Wavelet Tour of Signal Processing, 1998 (Academic Press, San Diego, California).

11 Li, D., Magnuson, D. S. K. and Jung, R. Non-stationary analysis of extracellular neural activity. Neurocomputing, 2000, 32-33, 1083-1093.

12 Li, D. and Jung, R. Tracking rhythmicity in nonstationary quasi-periodic biomedical signals using adaptive timevarying covariance. Computers in Biol. and Med., 2002, 32, 261-282.
13 Signal Processing Toolbox for Use with Matlab, Version 6, September 2000 (Mathworks Incorporated, Natick, MA).

14 Wavelet Toolbox for Use with Matlab, Version 6, September 2000 (Mathworks Incorporated, Natick, MA).

15 Measurement and evaluation of human exposure to vibration transmitted to the hand. BS 6842: 1987 (British Standards Institution, London).

16 MSC Series Multi-channel Conditioning Unit Instruction Manual, 1991 (Entran Devices Limited, Watford).

17 Digital Sony PC216A. Instrumentation-cassette recorder/ channel expansion unit manual, 1994.

18 TDS 210/220 Digital Real-time Oscilloscope User Manual, 1999 (Tektronix Incorporated, Wilsonville).

19 LMS Cada-X Fourier Monitor Manual, Revision 3.5.B, 1996 (LMS International, Leuvan).

20 Owen, K. and Coley, T. Automotive Fuels Reference Book, 2nd edition, 1995 (Society of Automotive Engineers, Warrendale, Pennsylvania). 